\title{
Cable Crosstalk Suppression in Resistive Sensor Array with 2-Wire S-NSDE-EP Method
}

\author{
JianFeng $\mathrm{Wu}^{1}$ and Lei Wang ${ }^{2}$ \\ ${ }^{1}$ Jiangsu Key Lab of Remote Measurement and Control, School of Instrument Science and Engineering, Southeast University, \\ Nanjing 210096, China \\ ${ }^{2}$ School of Automation, Nanjing Institute of Technology, Nanjing 211167, China \\ Correspondence should be addressed to JianFeng Wu; wjf@seu.edu.cn
}

Received 8 December 2015; Revised 26 January 2016; Accepted 28 January 2016

Academic Editor: Fernando Torres

Copyright (C) $2016 \mathrm{~J}$. Wu and L. Wang. This is an open access article distributed under the Creative Commons Attribution License, which permits unrestricted use, distribution, and reproduction in any medium, provided the original work is properly cited.

\begin{abstract}
With long flexible cables connected to the 1-wire setting non-scanned-driving-electrode equipotential (S-NSDE-EP) circuit, the resistive sensor array modules got flexibility in robotic operations but suffered from the crosstalk problem caused by wire resistances and contacted resistances of the cables. Firstly, we designed a new S-NSDE-EP circuit using two wires for every driving-electrode and every sampling-electrode to reduce the crosstalk caused by the connected cables in the 2D networked resistive sensor array. Then, an equivalent resistance expression of the element being tested (EBT) for this circuit was analytically derived. Then, the 1-wire S-NSDE-EP circuit and the 2-wire S-NSDE-EP circuit were evaluated by simulations. The simulation results show that the 2-wire S-NSDE-EP circuit, though it requires a large number of wires, can greatly reduce the crosstalk error caused by wire resistances and contacted resistances of the cables in the 2D networked resistive sensor array.
\end{abstract}

\section{Introduction}

Resistive sensor arrays were widely used in tactile sensing [1-8], light sensing [9], infrared sensing [10], and so forth. In robotic applications, long flexible cables were preferred for flexibility and limited space of the sensitive areas. With tested cables of lengths from $55 \mathrm{~mm}$ to $500 \mathrm{~mm}$ (as shown in Table 1), different modules of resistive sensor arrays were connected to the test circuits through the plugs and the sockets. Vidal-Verdú et al. $[1,3]$ designed and compared circuits of networked piezoresistive sensor arrays. Speeter [2] designed a flexible sensing system with $16 \times 16$ resistive taxels. Yang et al. [4] designed a $32 \times 32$ flexible array within a $160 \mathrm{~mm} \times 160 \mathrm{~mm}$ temperature and tactile sensing area. Zhang et al. [5] reported a $3 \times 3$ thin tactile force sensor array based on conductive rubber. Castellanos-Ramos et al. [6] reported a $16 \times 16$ tactile sensor array based on conductive polymers with screen-printing technology. Kim et al. [7] reported a flexible tactile sensor array with high performance in sensing contact force. Lazzarini et al. [8] reported a $16 \times 16$ tactile sensor array for practical applications in manipulation.
But cables had different wire resistances which increased with the increase of their lengths. Between the plugs of the connected cables and the sockets of the test circuits, there existed contacted resistances of tens of milliohms to several ohms varying with the variation of mechanical vibration and time. But new methods are still lacking, which can be used to suppress crosstalk caused by long cables.

For this purpose, we present a novel cable crosstalk suppression circuit based on a 2-wire method for the $2 \mathrm{D}$ networked resistive sensor arrays in the row-column fashion. This paper begins with an overview of the application fields of the 2D networked resistive sensor arrays. Secondly, a novel cable crosstalk suppression method will be proposed and its equivalent resistance expression of the element being tested (EBT) will be analytically derived. Then simulations will be implemented to evaluate this method with different parameters such as wire resistances and contacted resistances of the cables, the array size, the measurement range of the EBT, and the adjacent elements' resistances of $2 \mathrm{D}$ networked resistive sensor arrays. Finally, the results of experiments will be analyzed and conclusions for the method will be given. 
TABLE 1: Resistive sensor arrays with cables of different lengths.

\begin{tabular}{lcccc}
\hline Literature & Sensor & Array size of sensing elements & Cable length (mm) & Cable crosstalk \\
\hline$[1]$ & Polymer based FSR & $16 \times 9$ & $>55$ & $>60$ \\
{$[2]$} & FSR & $16 \times 16$ & $>70$ & Yes \\
{$[3]$} & FSR & $16 \times 16$ & $>70$ & Yes \\
{$[4]$} & Conductive rubber & $32 \times 32$ & $>95$ & Yes \\
{$[5]$} & Conductive rubber & $3 \times 3$ & $>100$ & Yes \\
{$[6]$} & Conductive polymer & $16 \times 16$ & $>100$ & Yes \\
{$[7]$} & Semiconductor strain gage & $5 \times 5$ & 500 & Yes \\
{$[8]$} & FSR & $16 \times 16$ & - & Yes \\
{$[9]$} & Light dependent resistor & $16 \times 16$ & & \\
\hline
\end{tabular}

\section{Principle Analyses}

In the row-column fashion, $2 \mathrm{D}$ resistive sensor arrays needed few wires but suffered from crosstalk caused by parasitic parallel paths. For suppressing crosstalk, many methods have been proposed and analyzed in literatures, such as the passive integrators method [3], the inserting diode method [11], the resistive matrix array method [12], the voltage feedback methods [2, 13-17], and the zero potential methods (ZPMs) [1, 3-10, 16-20]. Wu et al. have suppressed the crosstalk caused by the adjacent column elements and the adjacent row elements with the Improved Isolated Drive Feedback Circuit (IIDFC) [13] and the Improved Isolated Drive Feedback Circuit with Compensation (IIDFCC) [14]. Wu et al. have also proposed a general voltage feedback circuit model [15] for fast analyzing the performances of different voltage feedback circuits. D'Alessio has analyzed measurement errors in the scanning circuits of piezoresistive sensors arrays [16]. Saxena et al. $[18,19]$ have suppressed the crosstalk caused by the adjacent column elements with large number of opamps using the zero potential method. Roohollah et al. [20] have suppressed the crosstalk error caused by the input offset voltage and input bias current of the op-amp with a novel double-sampling technique. In these methods, the measurement accuracy of the EBT still suffered from cable crosstalk.

Liu et al. [17] defined the setting non-scanned-electrode zero potential (S-NSE-ZP) method, the setting non-scannedsampling-electrode zero potential (S-NSSE-ZP) method, and the setting non-scanned-driving-electrode zero potential (SNSDE-ZP) method for the zero potential methods, in which bipolar power sources were necessary for op-amps and analog digital converters (ADCs). In some circuits $[1,3]$, the reference voltages were not zero, so op-amps and ADCs with unipolar power sources, which were of less cost and were more convenient for use, could be used. So we defined those equipotential methods as the setting non-scanned-electrodeequipotential (S-NSE-EP) method, the setting non-scannedsampling-electrode-equipotential (S-NSSE-EP) method, and the setting non-scanned-driving-electrode-equipotential (SNSDE-EP) method. In this analysis, the S-NSDE-EP circuit was taken for example. Traditional S-NSDE-EP circuit of resistive networked sensor array in shared row-column fashion was shown as Circuit A in Figure 1(a). In Circuit A, the row electrodes and the column electrodes were used as the sampling electrodes and the driving electrodes, respectively. In Circuit $\mathrm{A}, R_{11}$ in the $M \times N$ resistive array was the element being tested (EBT); only one connected wire was used for every column and row electrode between the sensor array and the circuit; only one equal current $M: 1$ multiplexer was used between the current setting resistor $\left(R_{\text {set1 }}\right)$ and the row electrodes of the sensor module. On column electrodes of the circuit, 2:1 multiplexers had multiplexer switch resistances $\left(R_{s c} s\right)$; column wires had column resistances $\left(R_{L c} s\right)$ including column wire resistances and column contacted resistances. On row electrodes of the circuit, the equal current $M: 1$ multiplexers had multiplexer switch resistances $\left(R_{s r} s\right)$; row wires had row resistances $\left(R_{L r} s\right)$ including row wire resistances and row contacted resistances. Thus Circuit $\mathrm{A}$ had one row sampling op-amp, one $M: 1$ multiplexer, $N 2: 1$ multiplexers, and $M+N$ wires.

Under an ideal condition, all $R_{s c}$ s and all $R_{L c}$ s were omitted. Thus the voltage $\left(V_{c y 1}\right)$ on the column electrode of the EBT was equal to the feedback voltage $\left(V_{x y 1}\right)$, and the voltages on the nonscanned column electrodes were equal to the reference voltage $\left(V_{\text {ref } 1}\right)$. At the same time, all $R_{s r} \mathrm{~s}$ and all $R_{L r}$ s were omitted. Thus the voltage $\left(V_{e 1}\right)$ on the inverting input of the row sampling op-amp was equal to the voltage $\left(V_{r x 1}\right)$ on the row electrode of EBT. Under the effect of the ideal op-amp, $V_{e 1}$ was equal to $V_{\text {ref1 }}$ and the current $\left(I_{x y 1}\right)$ on the EBT was following the change of the current $\left(I_{\text {set } 1}\right)$ on $R_{\text {set } 1}$. As the voltages on the nonscanned column electrodes were equal to $V_{\text {ref1 }}$, the currents on the adjacent row elements of EBT were equal to zero. At the same time, the current on the inverting input of the ideal op-amp was omitted for its infinite input impedance, the current $\left(I_{x y 1}\right)$ on the EBT was equal to the current $\left(I_{\text {set } 1}=\left(V_{x y 1}-V_{\text {ref } 1}\right) / R_{x y 1}=V_{\text {ref } 1} / R_{\text {set } 1}\right)$ on $R_{\text {set } 1}$. Thus, $I_{\text {set } 1}$ and $I_{x y 1}$ were equal. As $V_{\text {ref } 1}$ and $R_{\text {set } 1}$ were known, $V_{x y 1}$ could be measured by ADC, so the equivalent resistance value $\left(R_{x y 1}\right)$ of the EBT in Circuit A could be calculated with the following:

$$
R_{x y 1}=\frac{\left(V_{x y 1}-V_{\text {ref1 }}\right) \times R_{\text {set1 }}}{V_{\text {ref1 }}} .
$$

But under the real condition as shown in Figure 1(b), $V_{c y 1}$ was not equal to $V_{x y 1}$ for $R_{s c}$ and $R_{L c}$, and $V_{e 1}$ was not equal to $V_{r x 1}$ for $R_{s r}$ and $R_{L r}$. The ideal feedback condition was destroyed by the row wires and the column wires, so extra measurement errors of the EBT existed. 


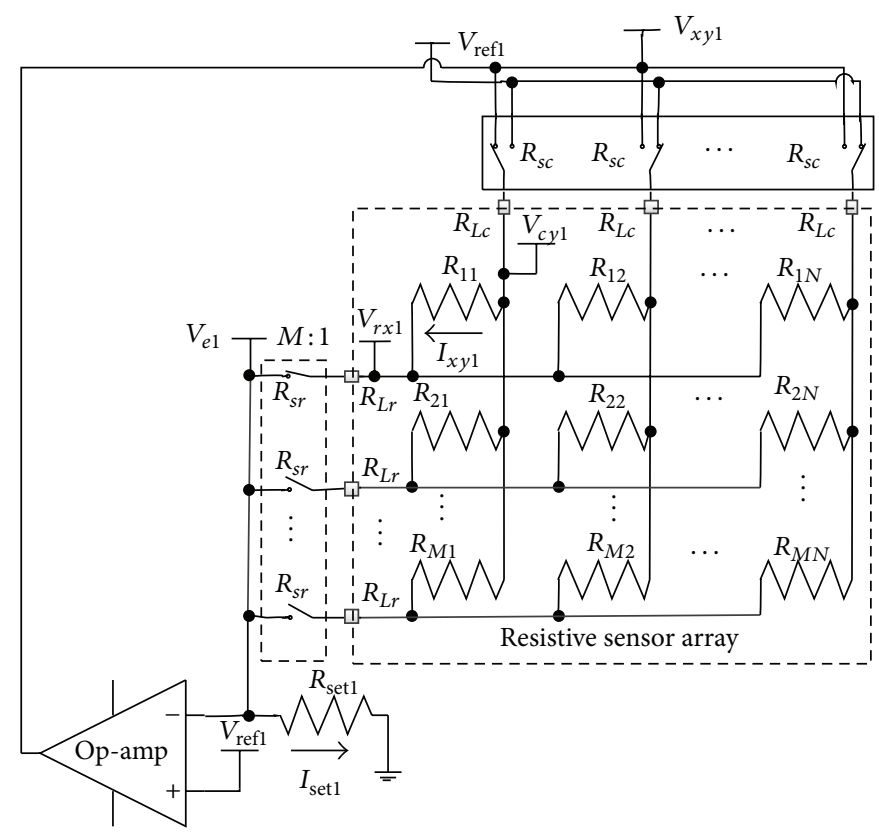

(a)

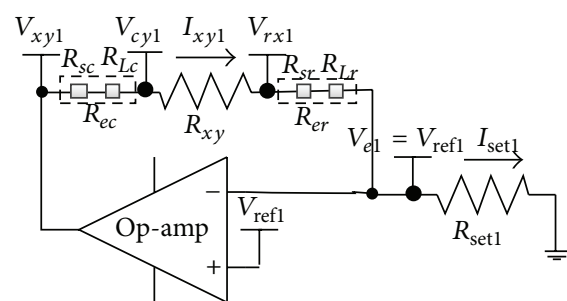

(b)

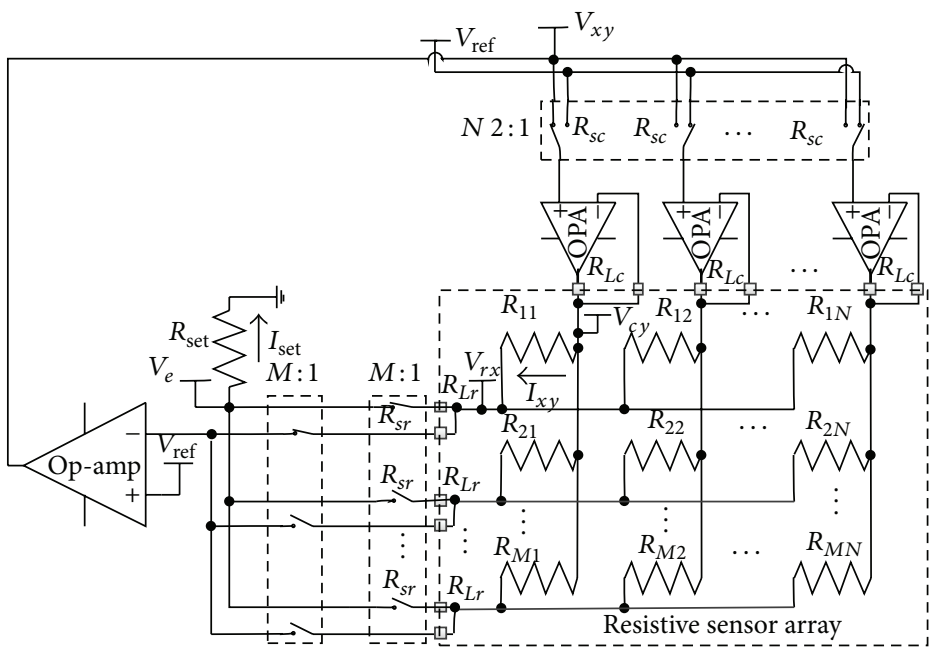

(c)

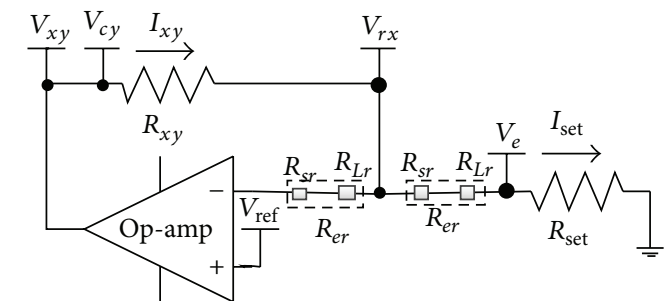

(d)

Figure 1: (a) 1-wire S-NSDE-EP circuit (Circuit A). (b) Simplified measurement circuit of 1-wire S-NSDE-EP circuit (Circuit B). (c) 2-wire S-NSDE-EP circuit (Circuit C). (d) Simplified measurement circuit of 2-wire S-NSDE-EP circuit (Circuit D).

For suppression cable crosstalk in the $2 \mathrm{D}$ networked resistive arrays, we proposed a 2 -wire equipotential method (Circuit C, as shown in Figure 1(c)). In Circuit C, we used two wires for every row electrode and every column electrode between the sensor module and the test circuit; also we used one column driving op-amp for every column electrode and one more equipotential $M: 1$ multiplexer between the row electrodes and the row sampling op-amp. Thus Circuit $\mathrm{C}$ had one row sampling op-amp, $N$ column driving op-amps, $N 2: 1$ multiplexers, two $M: 1$ multiplexers, and $2(M+N)$ connected wires.

Every column electrode in the sensor module was connected with the output of its column driving op-amp by one driving wire and it was also connected with the inverting input of its column driving op-amp by one driving sampling wire. The noninverting input of every column driving opamp was connected with the common port of its column $2: 1$ multiplexer; thus every noninverting input was connected with $V_{x y}$ or $V_{\text {ref }}$. The noninverting input of EBT's column driving op-amp was connected with $V_{x y}$ and the noninverting inputs of other column driving op-amps were connected with $V_{\text {ref }}$.

As the input impedance of every column driving opamp was much bigger than $R_{s c}$, the effect of $R_{s c}$ could be omitted. So the voltage on the noninverting input of every column driving op-amp was equal to the input voltage $\left(V_{x y}\right.$ or $V_{\text {ref }}$ ) of its $2: 1$ multiplexer. If the column driving op-amps had sufficient driving ability, the voltage on every column 
electrode was following the change of the voltage on the noninverting input of its column driving op-amp. So $V_{c y}$ was equal to $V_{x y}$, and the voltages on nonscanned column electrodes were equal to $V_{\text {ref }}$. Thus the crosstalk effect of $R_{L c}$ and $R_{s c}$ was suppressed.

By one equal current wire, every row electrode in the sensor module was connected with one channel of the equal current $M: 1$ multiplexer with its common port connected with $R_{\text {set }}$. In the equal current $M: 1$ multiplexer, only the row electrode of EBT was gated and all other nonscanned electrodes were suspended. So only the row electrode of the EBT was connected with $R_{\text {set }}$.

By one equipotential wire, every row electrode in the sensor module was also connected with one channel of the equipotential $M: 1$ multiplexer with its common port connected with the inverting input of the row sampling op-amp. In the equipotential $M: 1$ multiplexer, only the row electrode of EBT was gated and all other nonscanned electrodes were suspended. So only the EBT's row electrode was connected with the inverting input of the row sampling op-amp. From the output port of the EBT's column driving op-amp, the test current firstly flowed through the EBT, then it flowed through the row equal current wire, then it flowed through the equal current $M: 1$ multiplexer, and finally it flowed through $R_{\text {set }}$ to ground.

As the input impedance of the row sampling op-amp was much bigger than its series resistances such as the switch resistance of the equipotential $M: 1$ multiplexer, the wire resistance of the equipotential wire, and the contacted resistance, the voltage on the inverting input of the row sampling op-amp was equal to the voltage $\left(V_{r x}\right)$ of the EBT's row electrode.

Under the effect of the row sampling op-amp, the current $\left(I_{x y}\right)$ on the EBT followed the change of the current $\left(I_{\text {set }}\right)$ on $R_{\text {set }}$. As the input impedance of the row sampling opamp was much bigger than its parallel resistances such as $R_{s}$, $R_{s r}$, and $R_{L r}$, the leak current on the inverting input of the voltage feedback op-amp could be ommited. And the voltage on every nonscanned column electrode was equal to $V_{\text {ref }}$, which was also equal to $V_{r x}$. Thus the currents on the EBT's $(N-1)$ row adjacent elements were zero. So $I_{\text {set }}$ was equal to $I_{x y}$. The current with equal value also flowed through $R_{s r}$ and $R_{L r}$. As $R_{\text {set }}$ was known and $I_{\text {set }}$ was equal to $I_{x y}$, we could know $I_{x y}$ if the voltage $\left(V_{e}\right)$ on $R_{\text {set }}$ and the voltage $\left(V_{x y}\right)$ on the EBT were known. Thus we could get $R_{x y}$ of the EBT.

But $V_{e}$ was not equal to $V_{r x}$ for $R_{e r}$ (as shown in Figure 1(d)) which was the crosstalk caused by the row wire. Thus extra measurement error of the EBT was caused by it. From the above discussion, we could know that the currents on $R_{x y}, R_{\text {set }}$, and $R_{e r}$ had equal values. So we could use (2) to calculate $R_{x y}$ in Circuit C. We found that $R_{e r}$ did not exist in (2). As $V_{\text {ref }}$ and $R_{s}$ were known, $V_{x y}$ and $V_{e}$ could be measured by $\mathrm{ADC}$, so the equivalent resistance value $\left(R_{x y}\right)$ of the $\mathrm{EBT}$ in Circuit $C$ could be calculated with (2). Thus the crosstalk caused by the row wire was suppressed:

$$
R_{x y}=\frac{\left(V_{x y}-V_{\text {ref }}\right) \times R_{\text {set }}}{V_{e}} .
$$

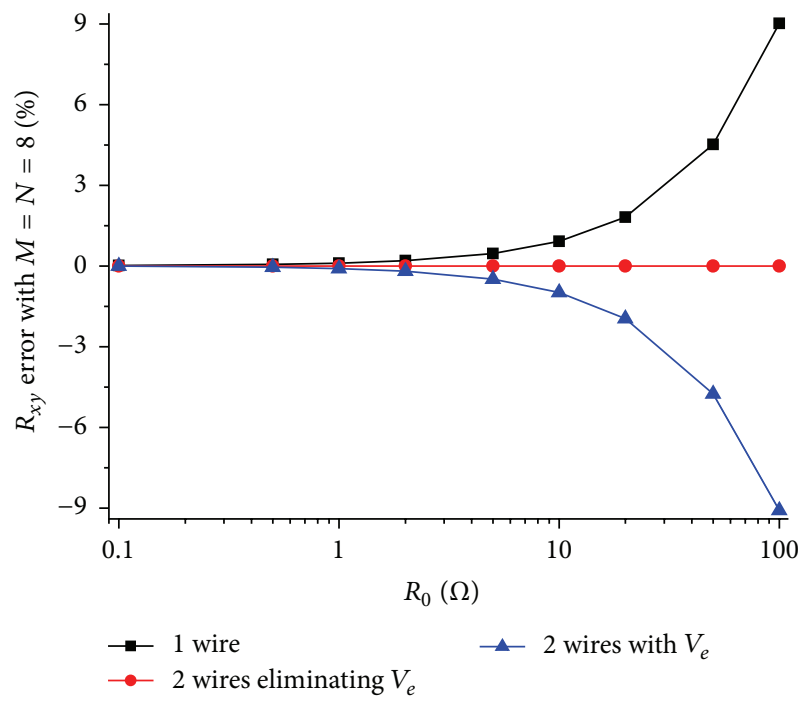

FIGURE 2: Effect of $R_{0}$ on the $R_{x y}$ errors in the 1-wire S-NSDE-EP circuit and the 2-wire S-NSDE-EP circuit where $M=N=8$.

From the above discussion, the 2-wire S-NSDE-EP method can depress the crosstalk caused by the row wires and the column wires such as $R_{s r} \mathrm{~s}, R_{L r} \mathrm{~s}, R_{s c} \mathrm{~s}$, and $R_{L c} \mathrm{~s}$.

\section{Simulation Experiments and Discussion}

To emulate the performance of our method, OP07 was selected as the macromodel of the op-amp (from the datasheet, the offset voltage, the bias current, the gainbandwidth, and the gain are equal to $75 \mu \mathrm{V}, 2.8 \mathrm{nA}, 0.60 \mathrm{MHz}$, and $126 \mathrm{~dB}$, resp.) in the simulations of National Instrument (NI) Multisim 12. In simulations, $V_{\text {ref }}$ was set at $0.1 \mathrm{~V}, R_{\text {set }}$ was set at $1 \mathrm{k} \Omega$, the positive voltage source of the op-amps was set at $9 \mathrm{~V}$, and the negative voltage source of the op-amps was set at $-6 \mathrm{~V}$.

3.1. $R_{0}$ Effect Simulation in NI Multisim. Cable resistance $\left(R_{0}, R_{0}=R_{e r}=R_{e c}\right)$ including the wire resistance and the contacted resistance affected the performance of the $2 \mathrm{D}$ networked resistive circuits. We investigated the effect of $R_{0}$ including wire resistance and contacted resistance on the 1wire S-NSDE-EP circuit and the 2-wire S-NSDE-EP circuit in NI Multisim. In simulations, we fixed some parameters including all elements in the resistive sensor array at $10 \mathrm{k} \Omega$ and $M$ and $N$ at 8 , and $R_{0}=R_{e r}=R_{e c}$ in sensor arrays varied synchronously with the same resistance value in $0.1 \Omega-100 \Omega$. The simulation results of the two circuits in NI Multisim 12 were shown in Figure 2. In the results, as shown in Figure 2, the deviation effect of $V_{e}$ caused by the row line and the row multiplexer was also considered.

From Figure 2, with $R_{0}$ varied from $0.1 \Omega$ to $100 \Omega, R_{x y}$ errors in the 1-wire S-NSDE-EP circuit showed a significant change (from $0.025 \%$ to $9.017 \%$ ) with an obvious positive increase coefficient, while $R_{x y}$ errors in the 2-wire S-NSDEEP circuit eliminating the deviation effect of $V_{e}$ showed a tiny change (from $-0.000 \%$ to $-0.003 \%$ ). But if the deviation 


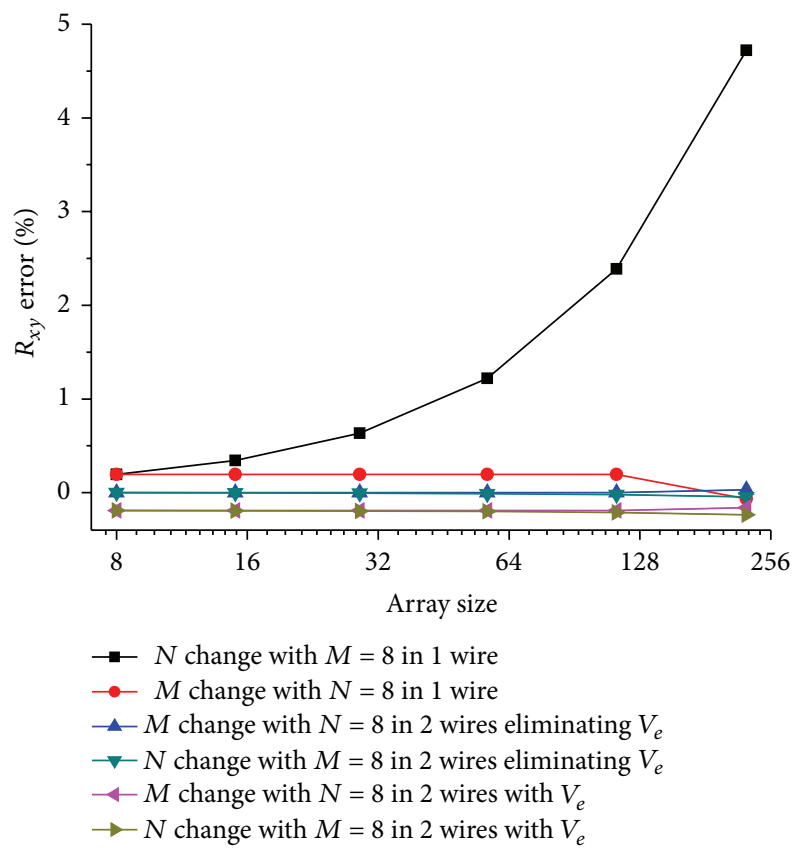

FIgURE 3: Array size effect on the $R_{x y}$ errors in the 1-wire S-NSDEEP circuit and the 2-wire S-NSDE-EP circuit where $R_{0}=2 \Omega$ and $R_{\text {other }}=10 \mathrm{k} \Omega$.

effect of $V_{e}$ was ignored, $R_{x y}$ errors in the 2-wire S-NSDEEP circuit with $V_{e}$ would be significant (from $-0.002 \%$ to $-9.083 \%$ ) as shown in Figure 2. Thus, the 2-wire S-NSDEEP circuit eliminating the deviation effect of $V_{e}$ has a better performance than the 1-wire S-NSDE-EP circuit when $R_{0}$ is varied from $0.1 \Omega$ to $100 \Omega$; the absolute $R_{x y}$ errors of the 2wire S-NSDE-EP circuit eliminating the deviation effect of $V_{e}$ are small enough to be negligible when $R_{0}$ is less than $100 \Omega$.

In the data of the simulation results, we also found the offset value of $V_{e}$ from $V_{\text {ref }}$ was varied from $0.19 \mathrm{mV}$ to $9.08 \mathrm{mV}$ with $R_{0}$ changing from $2 \Omega$ to $100 \Omega$.

3.2. Array Size Effect Simulation Experiment. Parameters of the array size such as the row number $(M)$ and the column number $(N)$ were proved to have effect on the performance of the $2 \mathrm{D}$ networked resistive sensor arrays [9-19]. We investigated the effect of $M$ and $N$ on the 1-wire S-NSDE-EP circuit and the 2-wire S-NSDE-EP circuit in NI Multisim. In simulations, we fixed some parameters including all elements in the resistive sensor array at $10 \mathrm{k} \Omega, M$ or $N$ at 8 , and $R_{0}$ at $2 \Omega$, and $N$ or $M$ was one number in $(8,15,29,57,113$, and 225$)$. The results of the array size effect on the 1-wire S-NSDE-EP circuit and the 2-wire S-NSDE-EP circuit were simulated in NI Multisim and the results were shown in Figure 3. In the results, as shown in Figure 3, the deviation effect of $V_{e}$ caused by the row line and the row multiplexer was also considered.

From Figure 3, with the increase of the column number, the $R_{x y}$ errors in the 1-wire S-NSDE-EP circuit had a positive coefficient (from $0.196 \%$ to $4.722 \%$ ) while the $R_{x y}$ errors in the 2-wire S-NSDE-EP circuit eliminating the deviation effect of $V_{e}$ had a negative coefficient (from $-0.000 \%$ to $-0.044 \%$ ).
But if the deviation effect of $V_{e}$ was ignored, we found a deviation of $R_{x y}$ errors (from $-0.191 \%$ to $-0.235 \%$ ) in the 2 wire S-NSDE-EP circuit with $V_{e}$ in Figure 3 . The absolute $R_{x y}$ errors in the 2 -wire S-NSDE-EP circuit eliminating the deviation effect of $V_{e}$ had been reduced significantly comparing with the absolute $R_{x y}$ errors in the 1-wire S-NSDEEP circuit.

From Figure 3, with the row number changed in the range from 8 to 113 , the $R_{x y}$ errors in both circuits changed little (from $0.196 \%$ to $0.194 \%$ for the 1 -wire S-NSDE-EP circuit, about $0.000 \%$ for the 2 -wire S-NSDE-EP circuit eliminating the deviation effect of $V_{e}$, about $-0.191 \%$ for the 2-wire SNSDE-EP circuit with $V_{e}$ ); but when the row number changed in the range from 113 to 225 , the $R_{x y}$ errors in both circuits changed clearly (from $0.194 \%$ to $-0.067 \%$ for the 1 -wire $S$ NSDE-EP circuit, from $0.000 \%$ to $0.032 \%$ for the 2 -wire SNSDE-EP circuit eliminating the deviation effect of $V_{e}$, from $-0.191 \%$ to $-0.159 \%$ for the 2 -wire S-NSDE-EP circuit with $\left.V_{e}\right)$. If every column driving op-amp had a sufficient current driving ability, the row number had less influence on the $R_{x y}$ errors in both circuits. In the data of the simulation results, we also found the offset value of $V_{e}$ from $V_{\text {ref }}$ was about $0.19 \mathrm{mV}$ with array size changed.

Thus, in the 2-wire S-NSDE-EP circuit eliminating the deviation effect of $V_{e}$, the influence of array size on the $R_{x y}$ error has been decreased greatly.

3.3. The Adjacent Elements Effect Simulation. In literatures [9-19], the adjacent elements played a significant role in affecting the measurement accuracy of the EBT. In simulations, we fixed some parameters including the resistance value of nonadjacent elements and all other adjacent elements at $10 \mathrm{k} \Omega, M$ and $N$ at 8 , and $R_{0}$ at $2 \Omega$. The resistance value of an adjacent element varied in the range from $0.1 \mathrm{k} \Omega$ to $1 \mathrm{M} \Omega$. The adjacent element could be an adjacent row element $\left(R_{\mathrm{adjr}}\right)$ or an adjacent column element $\left(R_{\mathrm{adjc}}\right)$. The simulation results of the 1-wire S-NSDE-EP circuit and the 2-wire S-NSDE-EP circuit in NI Multisim were shown in Figures 4-7.

From Figures 4-7, the $R_{x y}$ errors of the EBT of both circuits had negative coefficient when the resistance value of the EBT increased; the $R_{x y}$ errors of the EBT showed irregular variations when the resistances of the EBT was bigger than a certain value ( $\geq 30 \mathrm{k} \Omega$ for the 1-wire S-NSDE-EP circuit, $\geq 50 \mathrm{k} \Omega$ for the 2-wire S-NSDE-EP circuit). We found that the output voltages of the row sampling op-amp in both circuits were saturated for a bigger resistance value of the EBT. Under the same power source voltage, the measurement range of the 2-wire S-NSDE-EP circuit was bigger than that of the 1-wire S-NSDE-EP circuit.

From Figures 4-7, the $R_{x y}$ errors of the EBT with a bigger resistance value were susceptible to interference from by one $R_{\text {adjr }}$ or one $R_{\text {adjc }}$ with a smaller resistance value. In both circuits, the changes of the $R_{x y}$ errors for the change of one $R_{\text {adjr }}$ were bigger than the changes of the $R_{x y}$ errors for the change of one $R_{\mathrm{adjc}}$. With one $R_{\mathrm{adjr}}$ or one $R_{\text {adjc }}$ varied from $0.1 \mathrm{k} \Omega$ to $1 \mathrm{M} \Omega$, the changes of the $R_{x y}$ errors (with $R_{x y}$ at $30 \mathrm{k} \Omega$, from $-0.307 \%$ to $-0.048 \%$ for one $R_{\mathrm{adjc}}$ and from $-3.022 \%$ to $-0.051 \%$ for one $R_{\text {adjr }}$ ) in the 1 -wire S-NSDEEP circuit were significant, while those (with $R_{x y}$ at $50 \mathrm{k} \Omega$, 


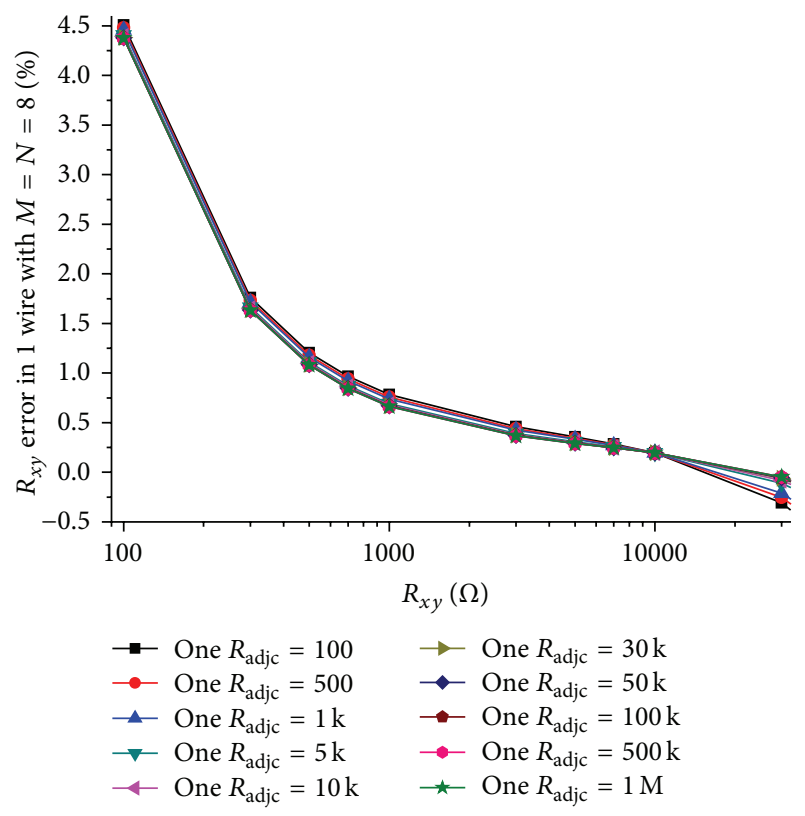

FIGURE 4: The $R_{\text {adjc }}$ effect on $R_{x y}$ errors in the 1-wire S-NSDE-EP circuit.

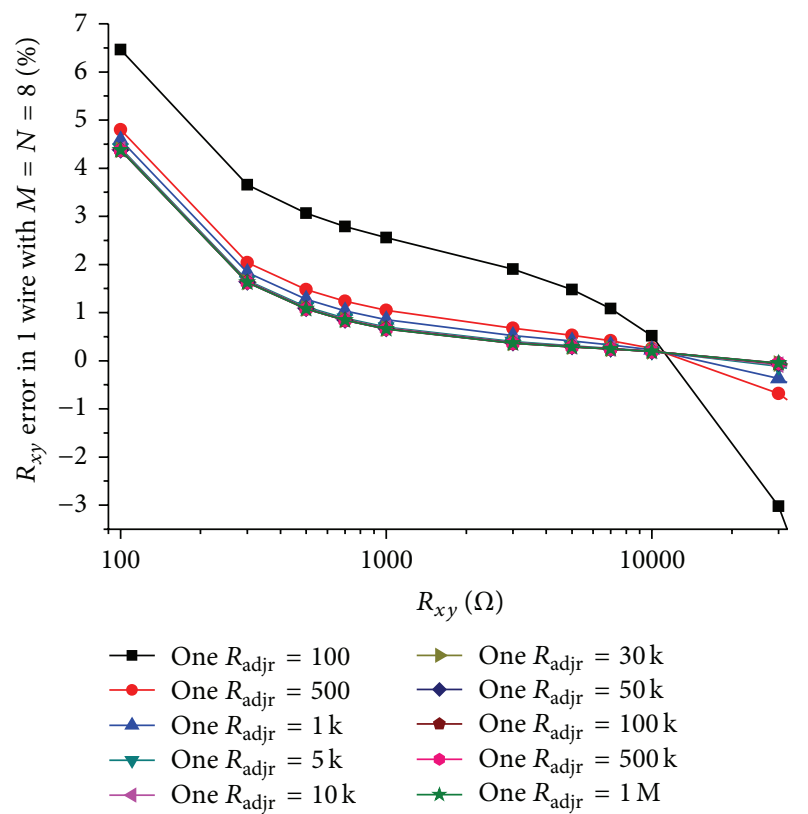

FIgURE 5: The $R_{\text {adjr }}$ effect on $R_{x y}$ errors in the 1-wire S-NSDE-EP circuit.

from $-0.006 \%$ to $-0.006 \%$ for one $R_{\text {adjc }}$ and from $-0.106 \%$ to $-0.005 \%$ for one $R_{\text {adjr }}$ ) in 2-wire S-NSDE-EP circuit were small. Thus, in the 2-wire S-NSDE-EP circuit, the influence of the adjacent elements on the $R_{x y}$ error has been decreased greatly.

3.4. The Op-Amp's Offset Voltage Effect Simulation. As many op-amps were used in the 2-wire S-NSDE-EP circuit, the offset voltages of the op-amps would affect the performance

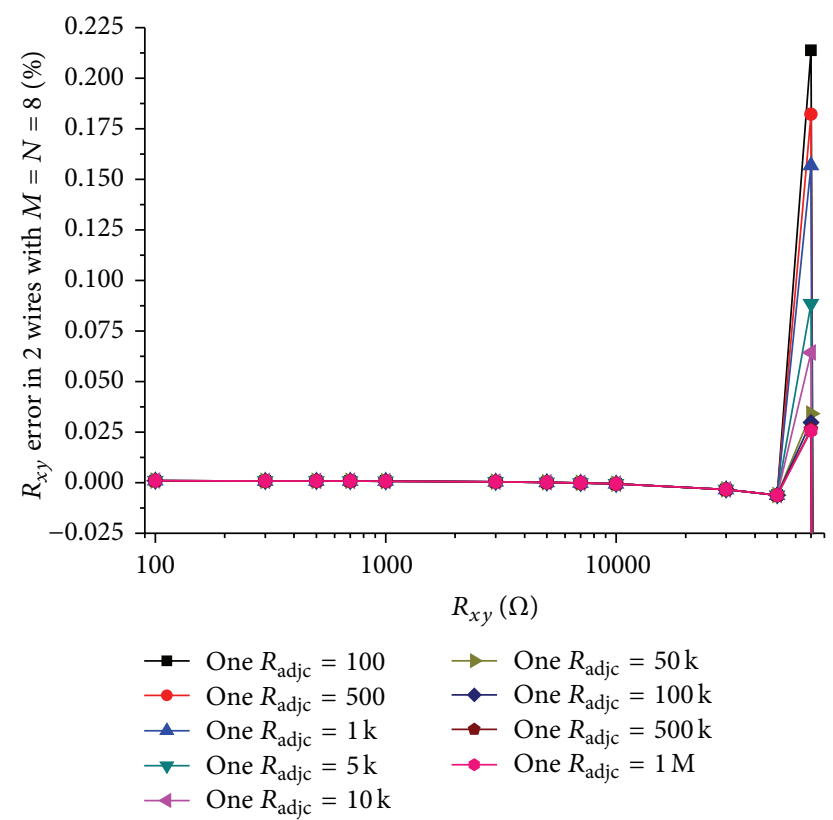

FIgURE 6: The $R_{\text {adjc }}$ effect on $R_{x y}$ errors in the 2-wire S-NSDE-EP circuit.

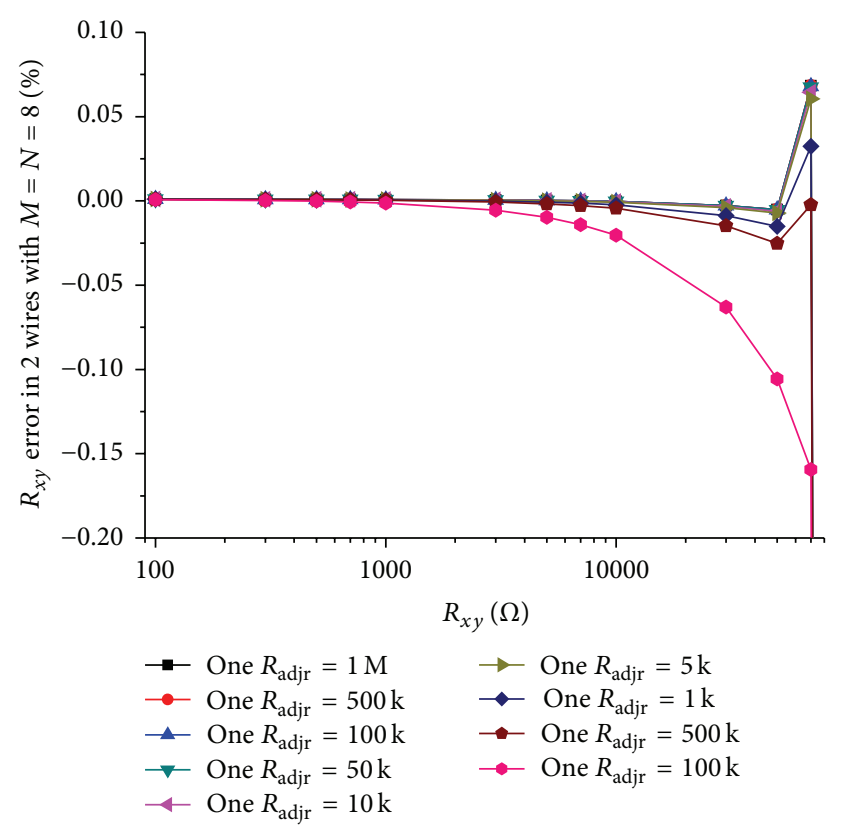

FIGURE 7: The $R_{\text {adjr }}$ effect on $R_{x y}$ errors in the 2-wire S-NSDE-EP circuit.

of the proposed circuit. In simulations, we fixed some parameters including the resistance value of all other row elements at $10 \mathrm{k} \Omega, M$ and $N$ at $8, R_{0}$ at $2 \Omega$, and all $R_{\text {ajcr }}$ at the same resistance value in $(100 \Omega, 300 \Omega, 1 \mathrm{k} \Omega$, and $10 \mathrm{k} \Omega)$. The offset voltages of the nonscanned column driving opamps varied synchronously with the same value in $(-75 \mu \mathrm{V}-$ $75 \mu \mathrm{V})$, and the 2-wire S-NSDE-EP circuit was simulated in NI Multisim and the results were shown in Figure 8. 


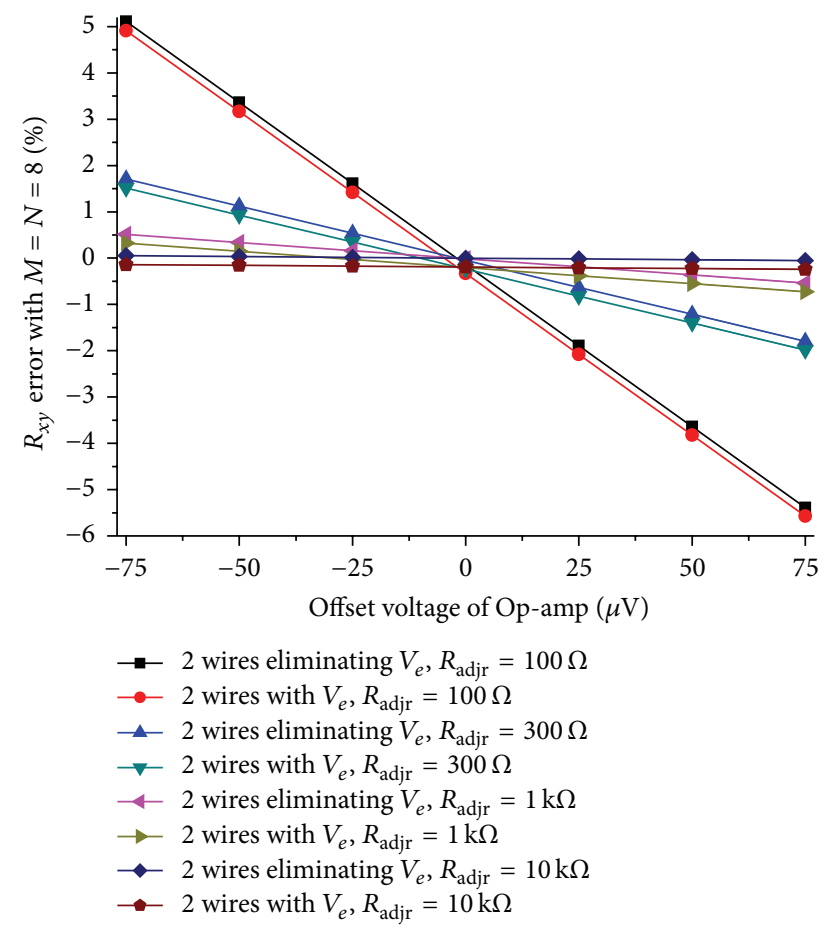

FIGURE 8: The op-amp's offset voltage effect on $R_{x y}$ errors in the 2wire S-NSDE-EP circuit.

From Figure 8, we found that the offset voltages of the opamps and the resistances of the row adjacent elements affected the 2-wire S-NSDE-EP circuit. The smaller these resistances were and the larger the offset voltage was, the larger the $R_{x y}$ error in the proposed circuit was.

3.5. The Op-Amp's Driving Capability Effect Simulation. The op-amp's driving capability affected the performance of the 2 -wire S-NSDE-EP circuit. The nonscanned elements' bypass effect on the EBT in the $2 \mathrm{D}$ resistive sensor array was obvious when the EBT had large resistance and all nonscanned elements had the small resistances. In the worst case, the EBT had the maximum resistance and all nonscanned elements had the minimum resistances [17]. In the experiments, we were about to simulate the op-amp's driving capability with all nonscanned elements of different fixed small resistances and the EBT of a large resistance. In simulations, we fixed some parameters including $M$ and $N$ at 8 and $R_{0}$ at $2 \Omega$ and all non-scanned elements at the same resistance value in $(100 \Omega$, $300 \Omega, 500 \Omega, 1 \mathrm{k} \Omega$, and $3 \mathrm{k} \Omega$ ). The resistance value of the EBT varied in the range from $0.1 \mathrm{k} \Omega$ to $60 \mathrm{k} \Omega$. The 2 -wire S-NSDE-EP circuit with the op-amp of OP07 was simulated in NI Multisim and the results were shown in Figure 9 and Table 2. Also the op-amps of OP07 $\left(I_{\text {short-circuit }}=30 \mathrm{~mA}\right)$ were replaced by the op-amps of $\mathrm{AD} 797\left(I_{\text {driving }}=50 \mathrm{~mA}\right)$, and the 2-wire S-NSDE-EP circuit was simulated.

From Figure 9 and Table 2, with the resistances of all nonscanned elements fixed, the 2-wire S-NSDE-EP circuit failed to work normally when the EBT's resistance exceeded certain values; with the minimum resistances of all nonscanned elements increased, the maximum resistance which could

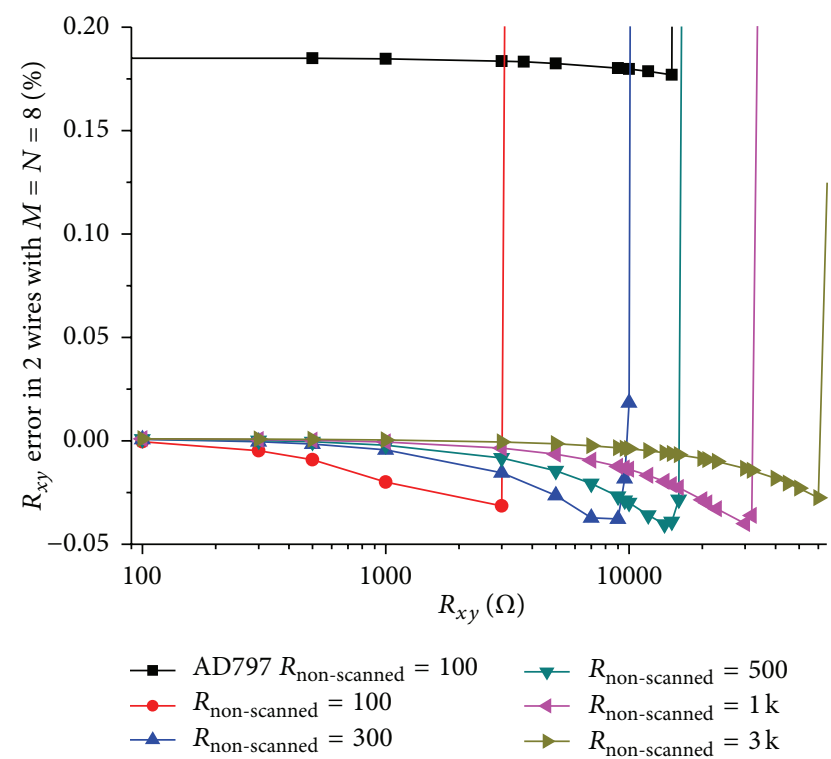

Figure 9: The op-amp's driving capability effect on $R_{x y}$ errors in the 2-wire S-NSDE-EP circuit.

TABLE 2: The EBT's maximum resistance of the 2-wire S-NSDE-EP circuit with its op-amp of OP07.

\begin{tabular}{lc}
\hline$R_{\text {non-scanned }}(\mathrm{k} \Omega)$ & The maximum resistance $(\mathrm{k} \Omega)$ \\
\hline 0.10 & 3.2 \\
0.30 & 9.6 \\
0.50 & 16.0 \\
1.00 & 32.0 \\
3.00 & 60.0 \\
\hline
\end{tabular}

be tested in the 2-wire S-NSDE-EP circuit increased; with a larger op-amp's driving capability, the 2-wire S-NSDE-EP circuit with its op-amp of AD797 had a larger measurement range.

3.6. Discussion. From the results in Figure 1, the 1-wire SNSDE-EP circuit had one voltage feedback op-amp, $N$ 2:1 multiplexers, one $M: 1$ multiplexers, and $M+N$ wires; the 2wire S-NSDE-EP circuit had one voltage feedback op-amp, $N$ column driving op-amps, $N$ 2:1 multiplexers, two $M: 1$ multiplexers, and $2(M+N)$ wires. Thus more components and more wires were used in the 2-wire S-NSDE-EP circuit.

From the results in Figure 2, the 2-wire S-NSDE-EP method was verified to be efficient in depressing the crosstalk caused by the row wires and the column wires such as $R_{s r}, R_{L r}$, $R_{s c}$, and $R_{L c}$. It should be noticed that all conductions were right under the assumption that the column driving op-amps had sufficient driving ability and the row sampling op-amp had very big input impedance on its inverting input.

From the results in Figures 3, 6, and 7, the 2-wire equipotential circuit was failed to work normally with too much big resistance value of the EBT. If the resistance of the adjacent elements in resistive sensor array was too small, the absolute $R_{x y}$ errors of the EBT would increase significantly. At the same time, if the row sampling op-amp did not have 
very big input impedance or the elements in resistive sensor array had very big resistance values for the row sampling opamp's input impedance, $I_{x y}$ would be not equal to $I_{\text {set. }}$. Thus the ideal work conditions were destroyed for the 2-wire SNSDE-EP circuit and the $R_{x y}$ error would be significant.

From the results in Figures 2, 3, and $8, V_{e}$ in the 2wire S-NSDE-EP circuit had a significant effect on the $R_{x y}$ error when the resistances such as the wire resistance, the contacted resistance, and the switch-on resistance of the equal current $M: 1$ multiplexer were large. Thus the deviation value of $V_{e}$, mainly caused by the connected cable and the equal current $M: 1$ multiplexers should be carefully considered in the 2-wire S-NSDE-EP circuit. In the proposed method, the deviation effect of $V_{e}$ had been eliminated and the 2-wire SNSDE-EP circuit with good performance was obtained. As the offset value of $V_{e}$ from $V_{\text {ref }}$ was varied from $0.19 \mathrm{mV}$ to $9.08 \mathrm{mV}$ with $R_{0}$ changing from $2 \Omega$ to $100 \Omega$, one more opamp was necessary for amplifying the signal of $V_{e}$ in the case of using an analog-digital converter with limited resolution in the 2-wire S-NSDE-EP circuit.

From the results in Figure 8, the offset voltages of the column driving op-amps had an obvious influence on the performance of the 2-wire S-NSDE-EP circuit, and the offset voltage's effect would be more obvious for the element being tested with its row adjacent elements of smaller resistance values. With the increase of the offset voltage, the $R_{x y}$ error increased. As the column number of the sensor array had accumulation influence on the conductance values of the row adjacent elements, it would enhance the effect of the offset voltage. Obviously, the offset voltage of the row sampling opamp had similar influence on the performance of the 2-wire S-NSDE-EP circuit. Thus in the practical circuit, the op-amps with smaller offset voltages were preferred. In the op-amp's offset voltage effect simulation experiments, the offset voltages of all nonscanned driving op-amps varied synchronously with the same value and their effect was obvious. But, in a practical circuit, the op-amps' offset voltages would be the uncertain values less than the offset voltage given in their datasheets and their effect would be weaker. In the 2-wire S-NSDE-EP circuit, the double-sampling technique [20] was also useful for eliminating the effect of those nonidealities of the op-amps such as the input offset voltage and the input bias current.

From the results in Figure 9 and Table 2, the op-amp's driving capability affected the measurement range of the 2wire S-NSDE-EP circuit; with the op-amp fixed, there was an approximate linear relation between the minimum resistance and the maximum resistance in the 2-wire S-NSDE-EP circuit. But the maximum resistance which could be tested in the 2-wire S-NSDE-EP circuit was also limited by the test current and the power source voltage. Thus the op-amps with large driving capability were preferred in the 2-wire S-NSDEEP circuit. But the op-amps with large driving capability always had a large offset voltage. So the contradiction between the driving capability affecting its measurement range and the offset voltage affecting its measurement accuracy should be balanced according to the test requirement.

For good performance of the IIDFC [13] and the IIDFCC [14], special compensated resistors with their resistances equal to their multiplexers' switch-on resistances are necessary. But the multiplexers' switch-on resistances may vary in the practical circuits, and the ideal performances of the IIDFC and the IIDFCC are difficult to realize. In the 2-wire S-NSDE-EP method, two wires for every row electrode and every column electrode between the sensor module and the test circuit, though it requires a large number of wires, are easier to achieve. The 2-wire S-NSDE-EP method's performance and its limitation have been verified by simulation experiments. Similar methods can also be used in the SNSSE-EP circuit and the S-NSE-EP circuit. But these should be verified in future practical application.

\section{Conclusion}

Firstly, a 2-wire S-NSDE-EP method of the 2D networked resistive sensor array was proposed. Secondly, the formula was given for the equivalent resistance expression of the element being tested in the networked sensor array by principle analyses. Then, the effects of some parameters on the measurement accuracy of the EBT were simulated with the National Instrument Multisim 12, the parameters including the wire resistances and the contacted resistances of long cables, the array size and the adjacent elements of the 2D resistive sensor array, and the offset voltages of the op-amps. The simulation results show that the 2-wire equipotential method was verified to be efficient in depressing the crosstalk caused by the row wires and the column wires such as $R_{s r}$, $R_{L r}, R_{s c}$, and $R_{L c}$; in the $2 \mathrm{D}$ networked resistive sensor array with the 2-wire S-NSDE-EP circuit, the influence of the adjacent column elements and the adjacent row elements on the measurement error of the element being tested has been reduced greatly. Finally, the factors which affected the performance of the 2-wire S-NSDE-EP circuit were discussed and the conclusion was given.

\section{Conflict of Interests}

The authors declare that there is no conflict of interests regarding the publication of this paper.

\section{Acknowledgment}

This study was supported by the Specialized Research Fund Program for the Doctoral Program of Higher Education (no. 20130092110060). This study was also supported by the Scientific Research Fund Project of Nanjing Institute of Technology (no. CKJB201405), the Open Fund of the Key Laboratory of Remote Measurement and Control Technology in Jiangsu Province (nos. YCCK201401 and YCCK201006), the National Major Scientific Equipment R\&D Project (Grant no. ZDYZ2010-2), and NSAF (no. U1230114).

\section{References}

[1] F. Vidal-Verdú, M. Jose Barquero, J. Castellanos-Ramos et al., "A large area tactile sensor patch based on commercial force sensors," Sensors, vol. 11, no. 5, pp. 5489-5507, 2011. 
[2] T. H. Speeter, "A tactile sensing system for robotic manipulation," The International Journal of Robotics Research, vol. 9, no. 6, pp. 25-36, 1990.

[3] F. Vidal-Verdú, Ó. Oballe-Peinado, J. A. Sánchez-Durán, J. Castellanos-Ramos, and R. Navas-González, "Three realizations and comparison of hardware for piezoresistive tactile sensors," Sensors, vol. 11, no. 3, pp. 3249-3266, 2011.

[4] Y.-J. Yang, M.-Y. Cheng, S.-C. Shih et al., "A $32 \times 32$ temperature and tactile sensing array using PI-copper films," The International Journal of Advanced Manufacturing Technology, vol. 46, no. 9, pp. 945-956, 2010.

[5] X. Zhang, Y. Zhao, and X. Zhang, "Design and fabrication of a thin and soft tactile force sensor array based on conductive rubber," Sensor Review, vol. 32, no. 4, pp. 273-279, 2012.

[6] J. Castellanos-Ramos, R. Navas-González, H. Macicior, T. Sikora, E. Ochoteco, and F. Vidal-Verdú, "Tactile sensors based on conductive polymers," Microsystem Technologies, vol. 16, no. 5, pp. 765-776, 2010.

[7] M.-S. Kim, H.-J. Shin, and Y.-K. Park, "Design concept of highperformance flexible tactile sensors with a robust structure," International Journal of Precision Engineering and Manufacturing, vol. 13, no. 11, pp. 1941-1947, 2012.

[8] R. Lazzarini, R. Magni, and P. Dario, "A tactile array sensor layered in an artificial skin," in Proceedings of the IEEE/RSJ International Conference on Intelligent Robots and Systems, Human Robot Interaction and Cooperative Robots, vol. 3, pp. 114-119, Pittsburgh, Pa, USA, August 1995.

[9] R. S. Saxena, R. K. Bhan, and A. Aggrawal, "A new discrete circuit for readout of resistive sensor arrays," Sensors and Actuators A: Physical, vol. 149, no. 1, pp. 93-99, 2009.

[10] R. S. Saxena, R. K. Bhan, C. R. Jalwania, and S. K. Lomash, "A novel test structure for process control monitor for uncooled bolometer area array detector technology," Journal of Semiconductor Technology and Science, vol. 6, no. 4, pp. 299312, 2006.

[11] D. Prutchi and M. Arcan, "Dynamic contact stress analysis using a compliant sensor array," Measurement, vol. 11, no. 3, pp. 197-210, 1993.

[12] L. Shu, X. Tao, and D. D. Feng, "A new approach for readout of resistive sensor arrays for wearable electronic applications," IEEE Sensors Journal, vol. 15, no. 1, pp. 442-452, 2015.

[13] J. F. Wu, L. Wang, and J. Q. Li, "Design and crosstalk error analysis of the circuit for the 2-D networked resistive sensor array," IEEE Sensors Journal, vol. 15, no. 2, pp. 1020-1026, 2015.

[14] J. F. Wu, L. Wang, J. Q. Li, and A. G. Song, "A novel crosstalk suppression method of the 2-D networked resistive sensor array," Sensors, vol. 14, no. 7, pp. 12816-12827, 2014.

[15] J. F. Wu, L. Wang, and J. Q. Li, "General voltage feedback circuit model in the two-dimensional networked resistive sensor array," Journal of Sensors, vol. 2015, Article ID 913828, 8 pages, 2015.

[16] T. D'Alessio, "Measurement errors in the scanning of piezoresistive sensors arrays," Sensors and Actuators A: Physical, vol. 72, no. 1, pp. 71-76, 1999.

[17] H. Liu, Y.-F. Zhang, Y.-W. Liu, and M.-H. Jin, "Measurement errors in the scanning of resistive sensor arrays," Sensors and Actuators A: Physical, vol. 163, no. 1, pp. 198-204, 2010.

[18] R. S. Saxena, R. K. Bhan, N. K. Saini, and R. Muralidharan, "Virtual ground technique for crosstalk suppression in networked resistive sensors," IEEE Sensors Journal, vol. 11, no. 2, pp. 432433, 2011.
[19] R. S. Saxena, S. K. Semwal, P. S. Rana, and R. K. Bhan, "Crosstalk suppression in networked resistive sensor arrays using virtual ground technique," International Journal of Electronics, vol. 100, no. 11, pp. 1579-1591, 2013.

[20] Y. Roohollah, A. Safarpour, and R. Lotfi, "An improvedaccuracy approach for readout of large-array resistive sensors," IEEE Sensor Journal, vol. 16, no. 1, pp. 210-215, 2015. 


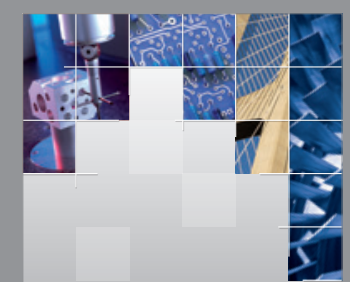

\section{Enfincering}
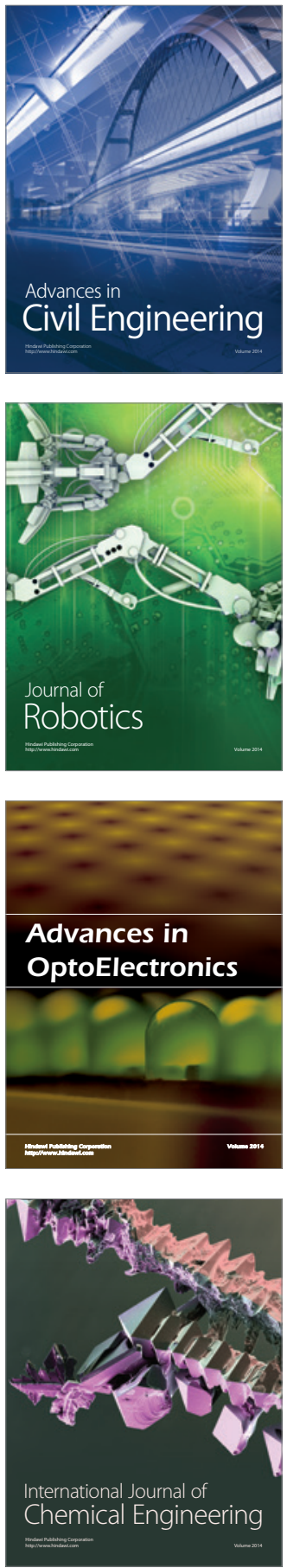

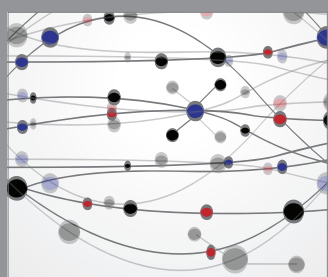

The Scientific World Journal

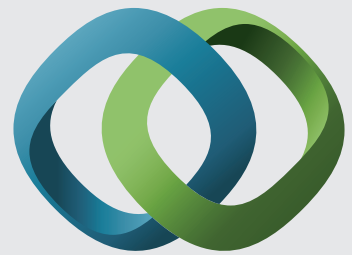

\section{Hindawi}

Submit your manuscripts at

http://www.hindawi.com
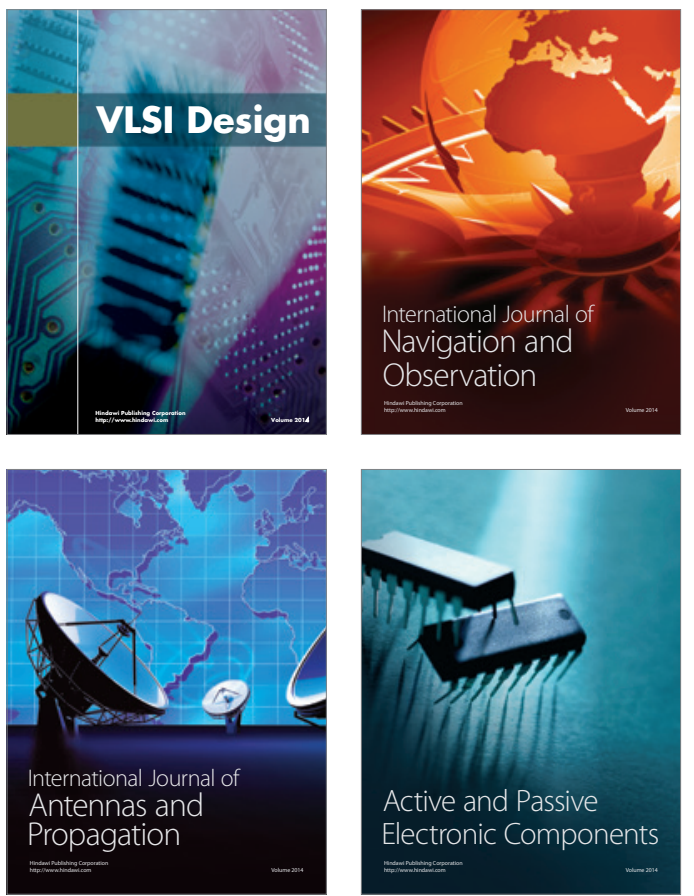
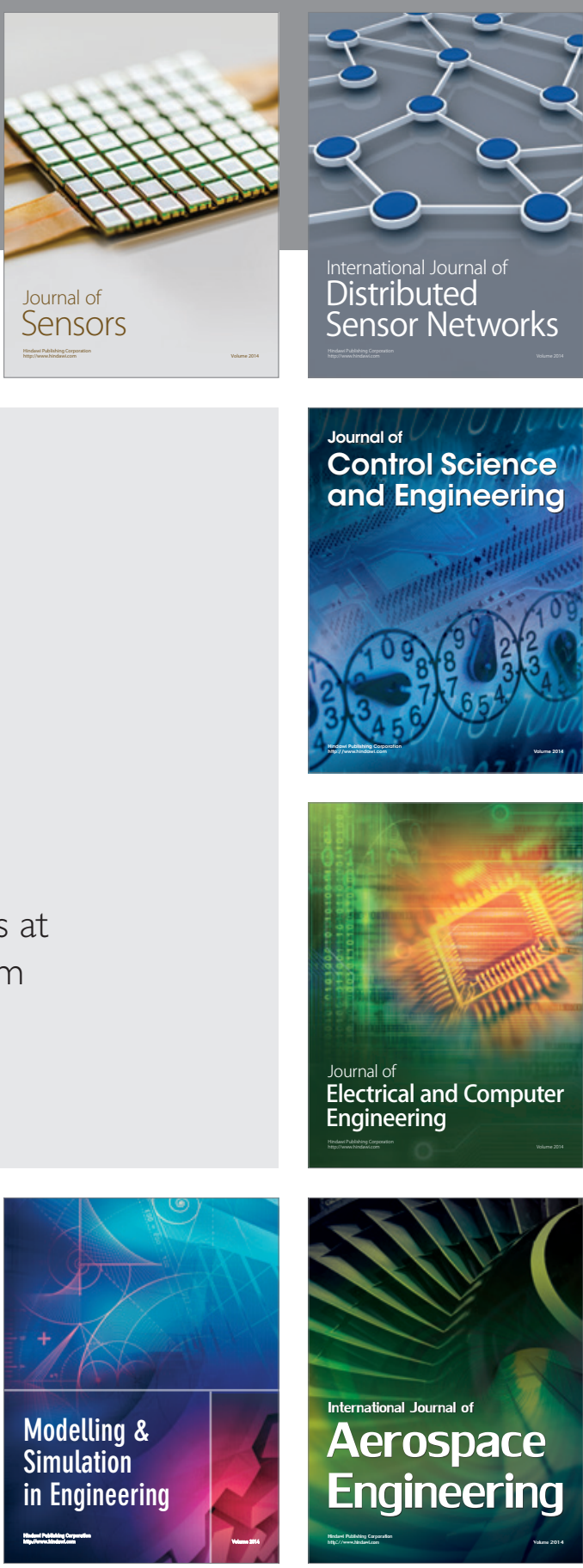

International Journal of

Distributed

Sensor Networks

Journal of

Control Science

and Engineering
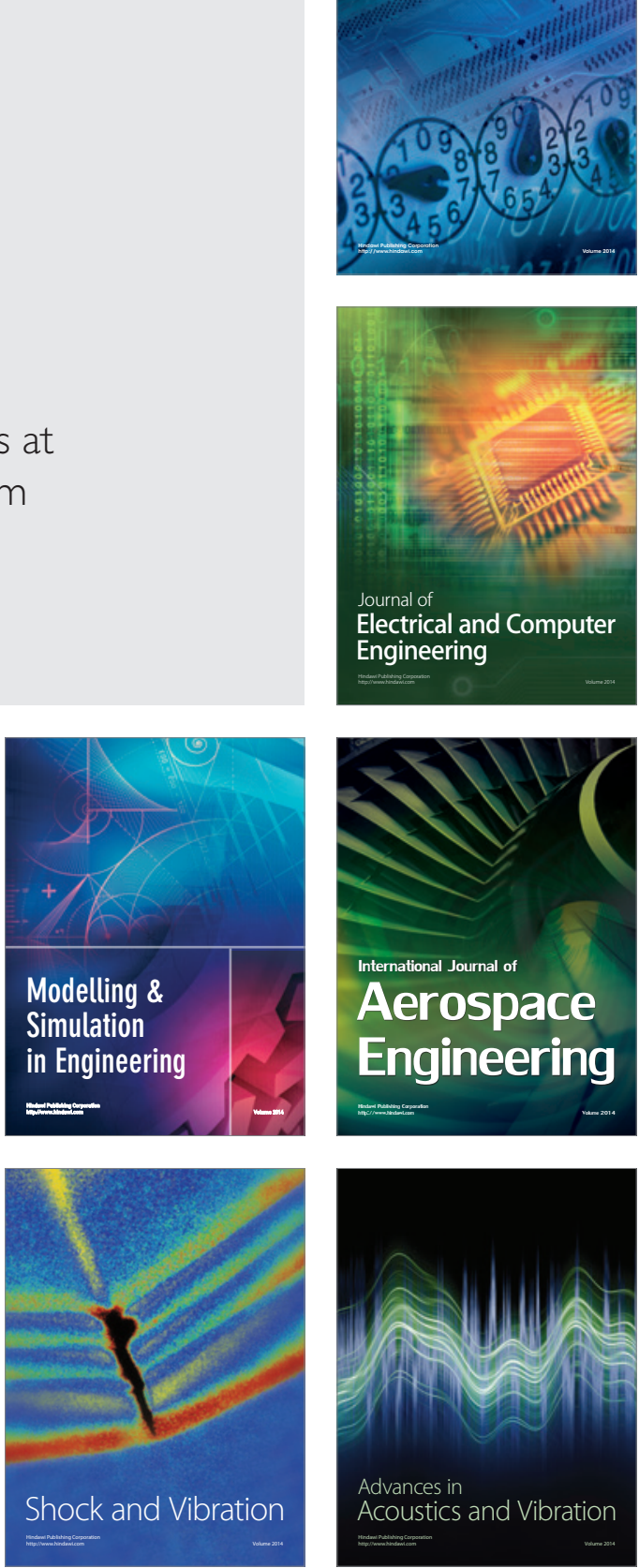